\title{
Homozygous SPAG6 variants can induce nonsyndromic asthenoteratozoospermia with severe MMAF
}

\author{
Chuan $\mathrm{Xu}^{1,2,3 \dagger}$, Dongdong Tang ${ }^{1,2,3 \dagger}$, Zhongmei Shao ${ }^{1,2,3 \dagger}$, Hao Geng ${ }^{1,4,5}$, Yang Gao ${ }^{1,4,5}$, Kuokuo Li $\mathrm{i}^{1,4,5}$, \\ Qing Tan ${ }^{1,4,5}$, Guanxiong Wang 1,4,5, Chao Wang ${ }^{1,4,5}$, Huan Wu ${ }^{1,4,5}$, Guanjian Li ${ }^{1,4,5}$, Mingrong LV 1,4, $^{*} \mathbb{D}_{\text {, }}$ \\ Xiaojin $\mathrm{He}^{1,2,3^{*}}$ and Yunxia Cao ${ }^{1,2,3^{*}}$
}

\begin{abstract}
Background: Multiple morphological abnormalities of the sperm flagella (MMAF) is a subtype of severe asthenoteratozoospermia with poorly understood genetic etiology. SPAG6 is a core axonemal component that plays a critical role in the formation of cilia and sperm flagella. Previous studies have reported that mutations in SPAG6 cause primary ciliary dyskinesia (PCD), but the association between SPAG6 gene variants and the MMAF phenotype has not yet been described.

Methods: We performed whole-exome sequencing (WES) in two unrelated Han Chinese men with MMAF. Sanger sequencing was used to validate the candidate variants. Routine semen analysis was carried out according to the WHO guidelines ( $5^{\text {th }}$ Edition). Sperm morphology was assessed using modified Papanicolaou staining. Scanning and transmission electron microscopy (S/TEM) was performed to observe the ultrastructural defects of the sperm flagella. Western blot analysis and immunofluorescence (IF) of spermatozoa were performed to examine the expression of SPAG6 protein. Assisted fertilization with intracytoplasmic sperm injection (ICSI) was applied.

Results: Two homozygous SPAG6 variants were identified by WES and Sanger validation in two patients with MMAF phenotype (F1 II-1: c.308C > A, p. A103D; F2 II-1: c. 585delA, p. K196Sfs*6). Semen analysis showed progressive rates of less than 1\%, and most of the spermatozoa presented MMAF by Papanicolaou staining. TEM revealed that the overall axonemal ultrastructure was disrupted and primarily presented an abnormal " $9+0$ " configuration. No other PCDrelated symptoms were found on physical examination and medical consultations, as well as lung CT screening. The level of SPAG6 protein was significantly decreased in the spermatozoa, and IF analysis revealed that SPAG6 staining was extremely weak and discontinuous in the sperm flagella of the two patients. Notably, F1 II-1 and his wife conceived successfully after undergoing ICSI.
\end{abstract}

\footnotetext{
*Correspondence: Ivmingrong2016@163.com; hxj0117@126.com; caoyunxia6@126.com

${ }^{\dagger}$ Chuan Xu, Dongdong Tang and Zhongmei Shao contributed equally to this work.

${ }^{1}$ Reproductive Medicine Center, Department of Obstetrics and Gynecology, the First Affiliated Hospital of Anhui Medical University, No 218 Jixi Road, Hefei 230022, Anhui, China

Full list of author information is available at the end of the article
} give appropriate credit to the original author(s) and the source, provide a link to the Creative Commons licence, and indicate if changes were made. The images or other third party material in this article are included in the article's Creative Commons licence, unless indicated otherwise in a credit line to the material. If material is not included in the article's Creative Commons licence and your intended use is not permitted by statutory regulation or exceeds the permitted use, you will need to obtain permission directly from the copyright holder. To view a copy of this licence, visit http://creativecommons.org/licenses/by/4.0/. The Creative Commons Public Domain Dedication waiver (http://creativecommons.org/publicdomain/zero/1.0/) applies to the data made available in this article, unless otherwise stated in a credit line to the data. 
Conclusions: Our research provides new evidence for a potential correlation between SPAG6 variants and the MMAF phenotype.

Keywords: MMAF, Gene, SPAG6, Asthenoteratozoospermia, ICSI

\section{Background}

Infertility affects $8-15 \%$ of couples who are trying to conceive, and has become a growing worldwide problem. Nearly half of all cases of infertility are attributed to male factors $[1,2]$. Asthenoteratozoospermia is one of the most common factors leading to male infertility, and is characterized by poor sperm motility and obvious sperm morphological abnormalities [3, 4]. As a subtype of asthenoteratozoospermia, multiple morphological abnormalities of the sperm flagella (MMAF) manifests as varied flagellar malformations, including short, coiled, bent, absent, and/or irregular flagella, and results in severely impaired sperm motility [5]. The absence of central microtubules is considered a hallmark of the MMAF phenotype [6].

Bi-allelic mutations in DNAH1 (MIM: 603,332) associated with MMAF were first described in 2014 [5]. To date, several genes responsible for MMAF have been identified, including DNAH8 (MIM: 603,337), SPEF2 (MIM:610,172), ARMC2(MIM:618,424), and WDR19 (MIM:608,151) [7-10]. Mutations in these genes can disorganize microtubule assembly, contributing to the abnormal formation of the sperm flagella. However, the reported monogenic causes can only account for approximately $35 \%$ to $60 \%$ of MMAF cases [11, 12 ], suggesting that this phenotype has strong genetic heterogeneity and that further genetic exploration is needed.

The sperm-associated antigen 6 (SPAG6; MIM: $605,730)$ gene encodes an axonemal protein that plays a critical role in axonemal structural integrity and function [13]. SPAG6 is primarily expressed in the lung and testis, and its variants are associated with multi-system dysfunctions involving cilia and flagella [14, 15], including primary ciliary dyskinesia (PCD). PCD is a rare multisystemic dysfunction caused by cilial motility malfunction. A recent study reported that bi-allelic mutations in SPAG6 are related to PCD, accompanied by recurrent respiratory tract infections and male infertility [14]. Similarly, the phenotype of Spag6 $6^{-1-}$ model mice is similar to that of some PCD-associated patients, including hydrocephalus and infertility resulting from a lack of motility related to ependymal cilia and sperm flagella [15]. These studies have demonstrated that mutations in SPAG6 are a genetic factor leading to syndromic asthenozoospermia, including PCD. However, the potential relationship between SPAG6 and nonsyndromic asthenoteratozoospermia characterized by the MMAF phenotype has not yet been described.

In this study, we performed whole-exome sequencing (WES) in two unrelated Han Chinese men affected with severe asthenoteratozoospermia, and homozygous variants in SPAG6 were identified in both cases. Notably, the two SPAG6-mutated probands consistently exhibited a typical MMAF phenotype and no other PCD-related symptoms. These results provide new evidence for a potential correlation between SPAG6 variants and nonsyndromic asthenoteratozoospermia characterized by the MMAF phenotype.

\section{Methods}

Subjects and clinical investigation

Two unrelated Han Chinese men from consanguineous families and diagnosed with primary infertility were recruited from the Reproductive Center of the First Affiliated Hospital of Anhui Medical University (Hefei, China), and were enrolled in the context of severe asthenoteratozoospermia with a representative MMAF phenotype. All subjects received detailed medical consultations and excluded other related risk factors, including abnormal chromosomal karyotypes, Y chromosome microdeletions, abnormal levels of sex hormones, radiotherapy, and chemotherapy. Physical examination revealed normal external genitalia and bilateral testicles, with no obvious abnormalities in the bilateral spermatic veins. Two healthy men with normal fertility and normal semen characteristics served as the control group. Peripheral whole blood from each individual was collected for subsequent genetic analysis. This research was reviewed and approved by the ethics board committee of the First Affiliated Hospital of Anhui Medical University, and all individuals provided written informed consent.

\section{WES and Sanger sequencing}

For WES and bioinformatic analysis, genomic DNA was extracted from whole peripheral blood samples and the exome was enriched using the SureSelect XT Human All Exon Kit (Agilent). Details of the study protocol have been published in our previous study [16]. Briefly, we extracted DNA from whole peripheral blood of the patients and sequenced with the MGISEQ-2000 platform. 
The data were mapped to the hg19 and GATK were used to call genetic variants. We annotated variants using allele frequency databases (the 1000 Genomes Project, gnomAD, and ExAC) and deleterious prediction tools. The variant with allele frequencies $>0.01$ were excluded. We focused on loss-of-function variants including stop gain/loss, frameshift insertion/deletion, splicing within two base pairs, and potentially deleterious missense variants that were predicted to be deleterious by two of the three tools (PolyPhen-2, SIFT, and Mutation Taster). The pathogenic variants in testis highly expressed genes were used for further analysis. We screened putative bi-allelic variants or X-linked variants of the patients according to the recessive or X-linked model of MMAF. Due to the consanguineous status and the low prevalence of MMAF, we mainly focused on autosomal homozygous variants or $\mathrm{X}$-linked variants. Sanger sequencing was used to validate the inheritance patterns. The pathogenic variants were also examined using WES for the respective female partners. Primers used for Sanger sequencing are listed in Supplementary Table 1.

\section{Semen analysis and sperm morphology}

Routine semen analysis was carried out using the Sperm Class Analyzer (SCA) 5.1 version software (Microptic, Spain), according to the World Health Organization guidelines (5th Edition) [17]. Sperm morphology was assessed using modified Papanicolaou staining. At least 200 spermatozoa from each participant were examined to assess defects in sperm flagella. The MMAF phenotype was classified into five categories: (1) absent, (2) short, (3) angulation, (4) coiled, and (5) irregular caliber flagella [5].

\section{Scanning and transmission electron microscopy}

For scanning electron microscopy (SEM) and transmission electron microscopy (TEM), sperm samples were collected and fixed with $2.5 \%$ glutaraldehyde at $4{ }^{\circ} \mathrm{C}$ for at least $2 \mathrm{~h}$. For SEM, after the dehydration step, the samples were dried chemically using hexamethyldisilazane, and then added dropwise to the specimen stubs. For TEM, samples were embedded in Epon, and ultrathin sections were cut and stained with uranyl acetate and lead citrate. Images were obtained with a Nova Nano 450 (Thermo Fisher, USA) and a Tecnai
G2 Spirit BioTWIN (FEI, USA) electron microscope, respectively.

\section{Western blotting and immunofluorescence staining}

Western blot analysis of spermatozoa was performed according to a previously described protocol [18]. The primary antibody was rabbit polyclonal anti-SPAG6 (HPA038440, Sigma, USA) antibody, and protein expression levels were normalized to that of beta-actin.

Immunofluorescence (IF) analysis was conducted to examine changes in the SPAG6 protein in the spermatozoa, according to our previously published protocol [19]. The primary antibodies were rabbit polyclonal anti-SPAG6 (HPA038440, Sigma, USA) and mouse monoclonal antiacetylated tubulin (5335S, Cell Signaling Technology, USA). Fluorescence images were captured using an LSM800 laser scanning confocal microscope (Zeiss, Germany).

\section{Assisted reproductive procedures}

Standard controlled ovarian hyperstimulation was performed according to our previous publication [20]. To enrich the motile spermatozoa, semen samples were processed by discontinuous density gradient centrifugation. After oocyte retrieval, mature oocytes and motile spermatozoa were selected for intracytoplasmic sperm injection (ICSI). All embryos formed after standard embryo culture were cryopreserved via vitrification for the following frozen-thawed cycles. After two months, either one or two viable blastocysts were thawed and transferred to the uterus of the female partner. Serum $\beta$-HCG levels were measured on day 14 after embryo transfer to determine biochemical pregnancy, and clinical pregnancy was defined as the presence of fetal heart activity in utero confirmed by B-ultrasound 30 days after embryo transfer.

\section{Results}

Two homozygous SPAG6 gene variants were identified in men with MMAF

To probe the genetic factors contributing to the MMAF phenotype, we analyzed the two probands by WES. Bioinformatics analysis was applied as mentioned in method section, and relevant or meaning mutations were remained (Supplementary Table 2). After bioinformatic filtering, the two probands were found to harbor homozygous variants of SPAG6, the only gene related to

(See figure on next page.)

Fig. 1 Variants of SPAG6 in the two patients with MMAF from two consanguineous families. A-B Pedigrees of the two families affected by the variants in SPAG6. The brown arrow and red dotted line show mutated locations in the validation of Sanger sequencing. $\mathbf{C}$ a schematic diagram of mutated positions occurred in the SPAG6 protein. The mutated positions of SPAG6 are conserved among species. The green boxes indicate the armadillo-type repeats domains of SPAG6 protein. WT, wild type; M, SPAG6 mutations; MMAF: multiple morphological abnormalities of the sperm flagella 


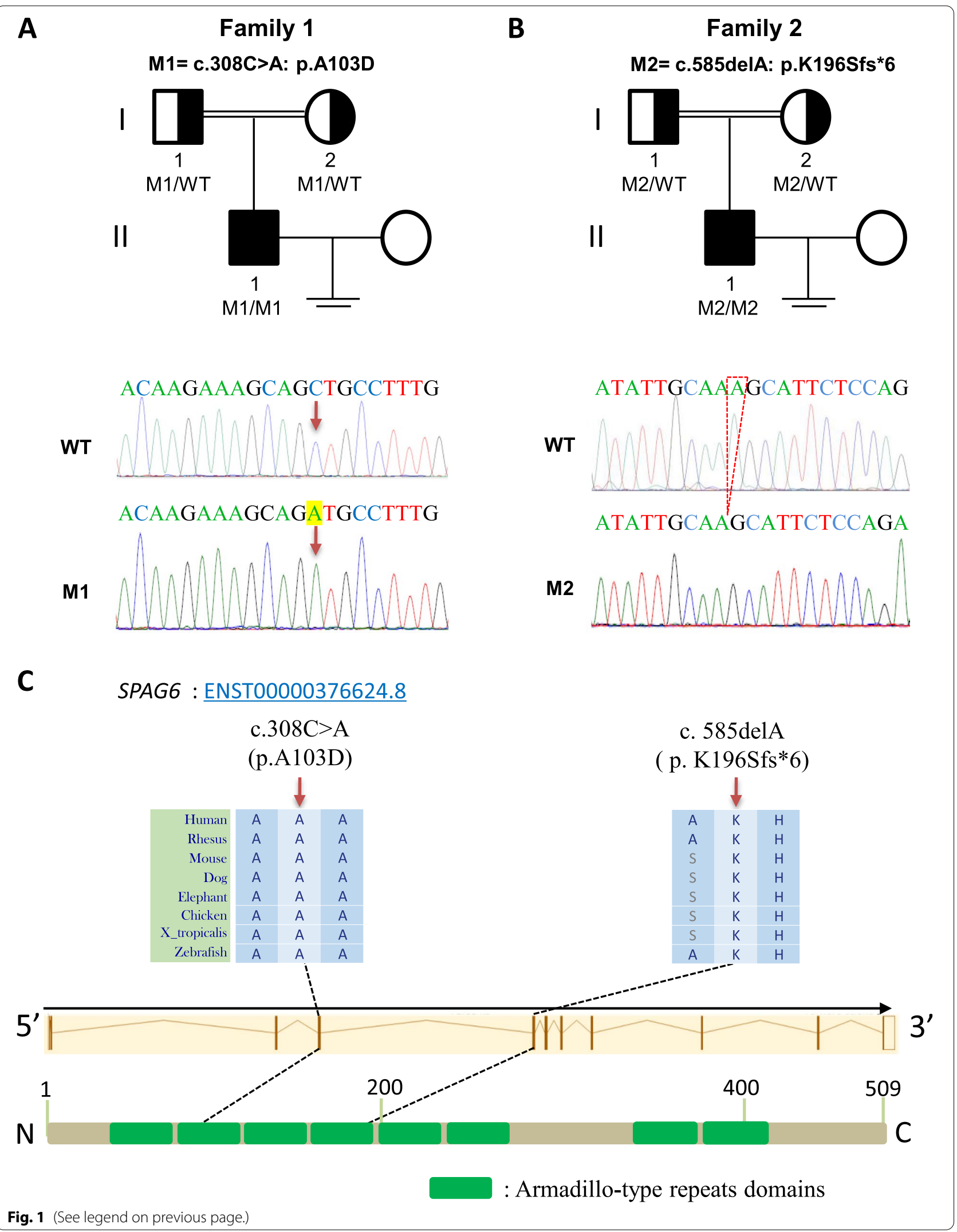


Table 1 Genetic information of SPAG6 variants of the patients

\begin{tabular}{lll}
\hline Subject & F1 II-1 & F2 II-1 \\
\hline cDNA mutation & c.308C >A & c. 585delA \\
Exon & Exon 4 & Exon 5 \\
$\begin{array}{l}\text { Mutation type } \\
\text { Protein alteration }\end{array}$ & nonsynonymous & Frameshift deletion \\
Allele frequency in human population & p. Lys196Serfs*6 \\
1KGP & NA & NA \\
EXAc_all & NA & $8.25 \times 10^{-6}$ \\
gnomAD & NA & $4.07 \times 10^{-6}$ \\
Deleterious prediction & & \\
SIFT & D & NA \\
PolyPhen-2 & $D$ & NA \\
Mutation Taster & D & NA \\
\hline
\end{tabular}

RefSeq accession number of SPAG6 is NM_ 012,443.4

Abbreviations: 1KGP 1000 Genomes Project, ExAc_all all the data of Exome Aggregation Consortium gnomAD the Genome Aggregation Database, $D$ disease-causing, NA not available sperm flagellar function that met the screening conditions. Following Sanger sequencing, homozygous SPAG6 variants were identified in two individuals and inherited from their heterozygous parental carriers. The details are summarized in the Fig. 1 and Table 1.

\section{Sperm analysis and clinical characteristics of men carrying SPAG6 variants}

Sperm parameters are summarized in Table 2. Semen analysis of the patients showed severe asthenoteratozoospermia with progressive motility rates of less than $1 \%$, while the semen volume, sperm concentration, and sperm vitality were normal. Clinical examinations were also performed. None of the patients suffered from PCD-related symptoms, such as chronic bronchitis, recurrent respiratory tract interference, otitis media, and visceral inversion (Supplementary Table 3). Lung CT imaging further confirmed normal results in both cases (Supplementary Fig. 1). The MMAF phenotype in the spermatozoa was examined

Table 2 Semen parameters and sperm morphology of men harbouring homozygous SPAG6 variants

\begin{tabular}{|c|c|c|c|c|c|}
\hline \multirow{3}{*}{$\begin{array}{l}\text { Subject } \\
\text { Age } \\
\text { Semen parameter }\end{array}$} & \multicolumn{2}{|l|}{ F1 II-1 } & \multicolumn{2}{|l|}{ F2 II-1 } & \multirow[t]{3}{*}{ Reference Values } \\
\hline & \multicolumn{2}{|l|}{29} & \multicolumn{2}{|l|}{26} & \\
\hline & Sample 1 & Sample 2 & Sample 1 & Sample 2 & \\
\hline Semen volume $(\mathrm{mL})$ & 2.2 & 3.5 & 3.4 & 3.6 & $>1.5$ \\
\hline Concentration $\left(10^{6} / \mathrm{mL}\right)$ & 107.0 & 63.9 & 16.8 & 27.4 & $>15.0$ \\
\hline Motility (\%) & 2.0 & 2.7 & 0 & 0 & $>40.0$ \\
\hline Progressive motility (\%) & 0.3 & 0.5 & 0 & 0 & $>32.0$ \\
\hline Viability (\%) & 66 & NA & 61 & NA & $>58.0$ \\
\hline \multicolumn{6}{|l|}{ Sperm Morphology } \\
\hline Normal flagella (\%) & 3.2 & & 4.1 & & $>23.0$ \\
\hline Absent flagella (\%) & 4.5 & & 7.1 & & $<5.0$ \\
\hline Short flagella (\%) & 55.3 & & 47.6 & & $<1.0$ \\
\hline Coiled flagella (\%) & 32.5 & & 39.2 & & $<17.0$ \\
\hline Angulation (\%) & 3.5 & & 1.5 & & $<13.0$ \\
\hline Irregular caliber (\%) & 1.0 & & 0.5 & & $<2.0$ \\
\hline
\end{tabular}

Abbreviations: NA Not applicable

a Reference Values according to the WHO (2010) criteria; Bold characters represent abnormal values

(See figure on next page.)

Fig. 2 Ultrastructural defects in spermatozoa from two individuals carrying SPAG6 variants. A Scanning electron microscopy of the spermatozoa from a healthy control and F1 II-1. (i) normal morphology of spermatozoa from a healthy control man; (ii-v) scanning electron microscopy showed the multiple abnormalities of the sperm flagella from F1 II-1 individual, including short(ii), coiled (iii, iv), and absent(v). Scale bar: $5 \mu$ m. B transmission electron microscopy of the sperm flagella from a healthy control and two patients. (i-iii) cross-sections of the sperm flagella in a healthy control, including mid-piece, principal piece and end-piece, show the typical " $9+2$ " axonemal structure and peri-axoneme structure. (iv-vi) and (vii-ix) axonemal cross-sections of the sperm flagella from F1 II-1 and F2 II-1, respectively. The overall axonemal ultrastructure was disrupted, and primarily presented an abnormal " $9+0$ " configuration. Of these, the lack of the central microtubules was the main defect observed in sperm flagella from two probands, peripheral microtubule doublets and outer dense fibers were also translocated and disorganized. Scale bar: 200 nm. Abbreviations: CP, central pair of microtubules (blue triangles); MT, peripheral microtubule doublets (yellow triangles); ODF, outer dense fiber (red triangles); 

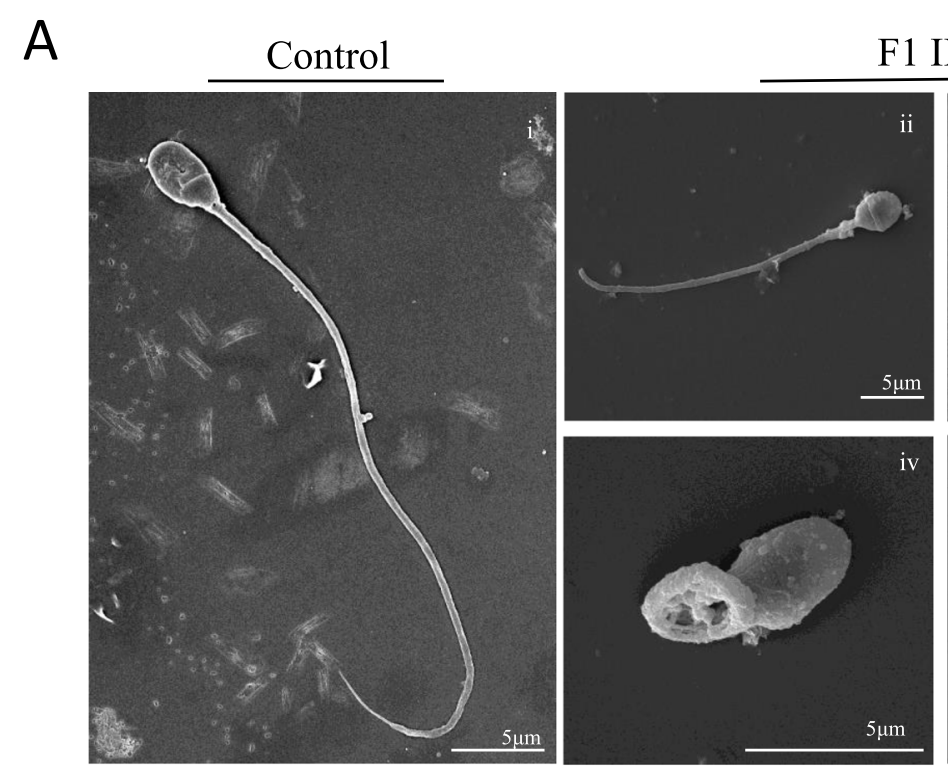

F1 II-1

B
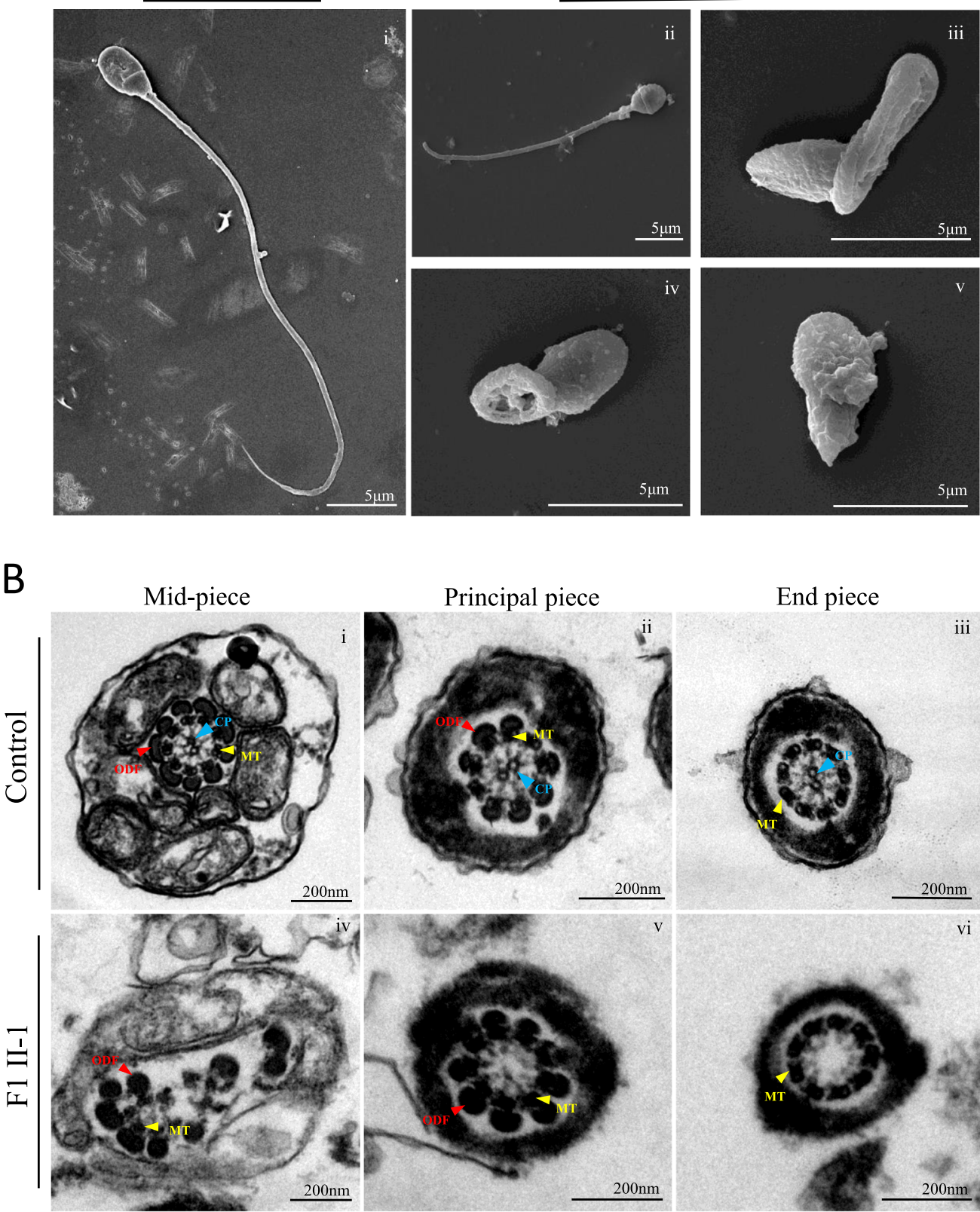

End piece
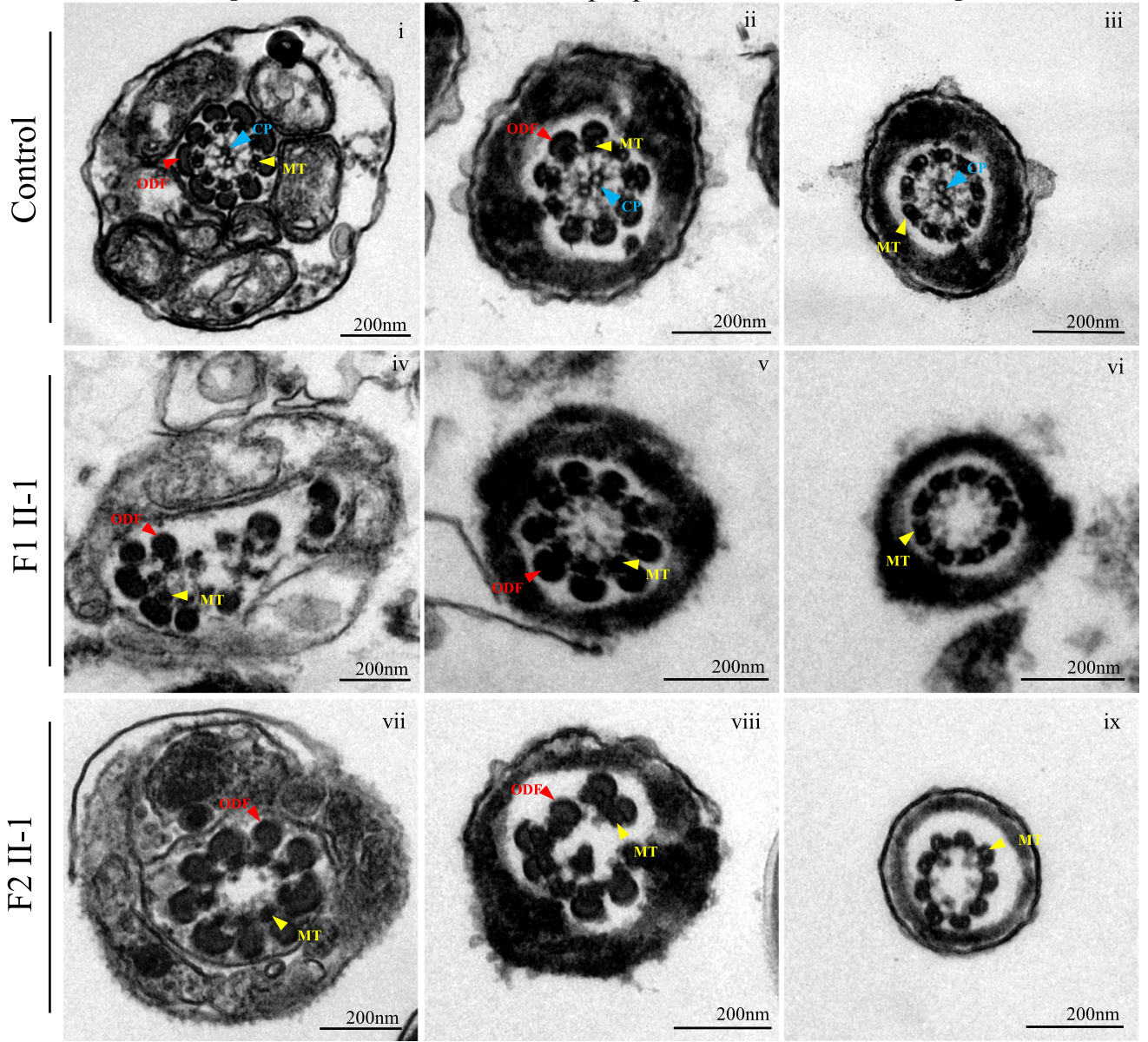

ix

Fig. 2 (See legend on previous page.) 

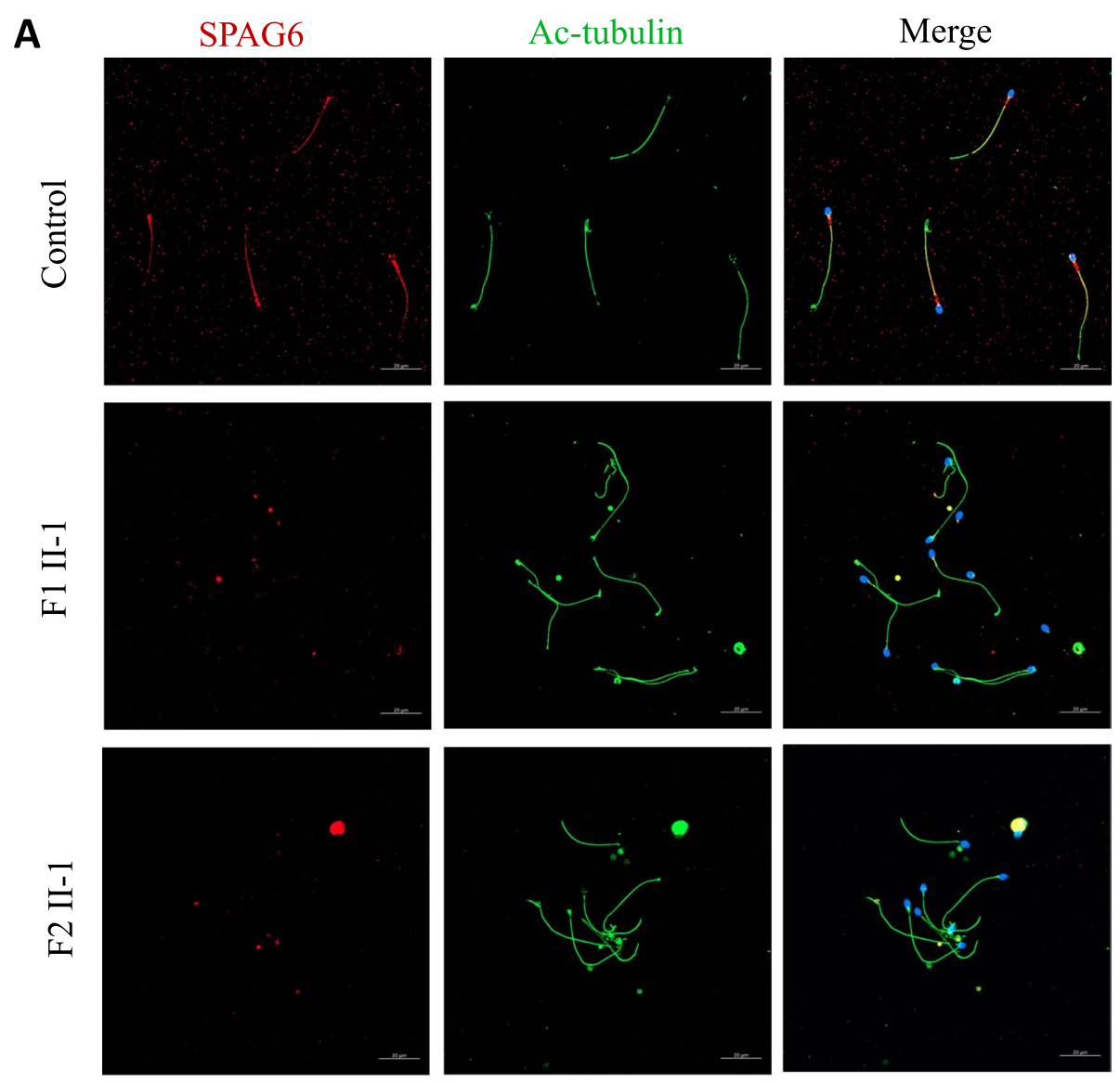

B

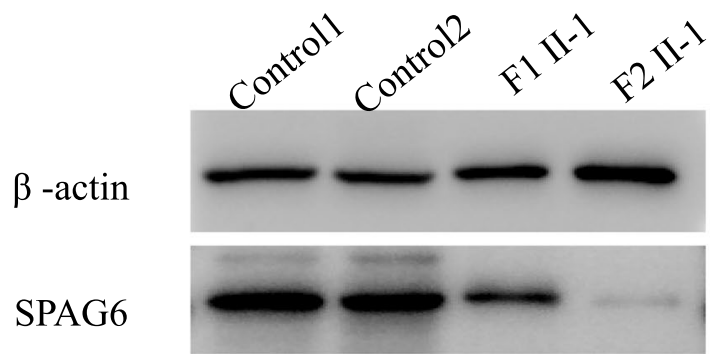

Fig. 3 Lower expression of SPAG6 in spermatozoa from men harboring SPAG6 variants. A Immunofluorescence analysis: SPAG6 staining (red) was located along entire the sperm flagella from a normal control, while SPAG6 staining was extremely weak and discontinuous in the sperm flagella from F1 II-1 and F2 II-1. The anti-acetylated tubulin staining (green) was used as a flagellar maker. Scale bar: 20 m. B SPAG6 protein levels were determined using western blotting in spermatozoa from F1 II-1, F2 II-1 and two healthy controls. Beta-actin was used as loading control

using light microscopy. According to the Papanicolaou staining method, most of the spermatozoa presented multiple flagellar malformations, including absent, short, coiled, angulation, and irregular flagella. Notably, short and coiled flagella were most frequently observed in the spermatozoa of the two patients.

To further reveal ultrastructural defects in the flagella, we also used S/TEM to analyze the spermatozoa from patients and controls. According to SEM, the spermatozoa of the patients primarily presented short and coiled flagella (Fig. 2A). For TEM, over 50 random flagellar cross-sections were observed for each SPAG6mutated proband to observe the microtubule assembly in the sperm flagella. In contrast to the typical " $9+2$ " configuration that was observed in the normal controls, the overall axonemal ultrastructure was disrupted, and primarily presented an abnormal " $9+0$ " configuration in sperm flagella of both patients. Of these, the lack of the 
central microtubules was the main defect observed in sperm flagella, additionally, peripheral microtubule doublets and outer dense fibers were also translocated and disorganized (Fig. 2B).

\section{Expression of SPAG6 was attenuated in spermatozoa from men with MMAF}

To further investigate the pathogenicity of the identified homozygous SPAG6 variants, expression of SPAG6 protein was analyzed in spermatozoa from the controls and two cases. The western blot showed an intense band at approximately $55 \mathrm{kDa}$ for the full-length SPAG6 protein in the normal control, and the intensity of this band was dramatically reduced in the two probands (Fig. 3B). The localization and expression of SPAG6 protein in spermatozoa was also detected by IF staining. SPAG6 staining was localized along the entire flagella of sperm from the controls, and normal axonemes were visible via staining of acetylated$\alpha$-tubulin. In contrast, SPAG6 staining was extremely weak and discontinuous in sperm flagella in both of the MMAF cases (Fig. 3A).

\section{ICSI outcomes}

ICSI treatment was performed due to male factor infertility in the two couples corresponding to the cases in the present study. All blastocysts were cryopreserved after standard embryo culture. Two months later, F1 II-1 and his partner successfully conceived after the transfer of one frozen-thawed embryo (Fig. 4). The F2 II-1 partner is currently waiting for embryo transfer. This result suggests that patients carrying SPAG6 variants have a good prognosis for ICSI. The ICSI outcomes of the two couples are summarized in Supplementary Table 4.

\section{Discussion}

Two patients with severe asthenoteratozoospermia harboring homozygous SPAG6 variants were identified. The two cases presented a typical MMAF phenotype without other PCD-related symptoms. The expression level of
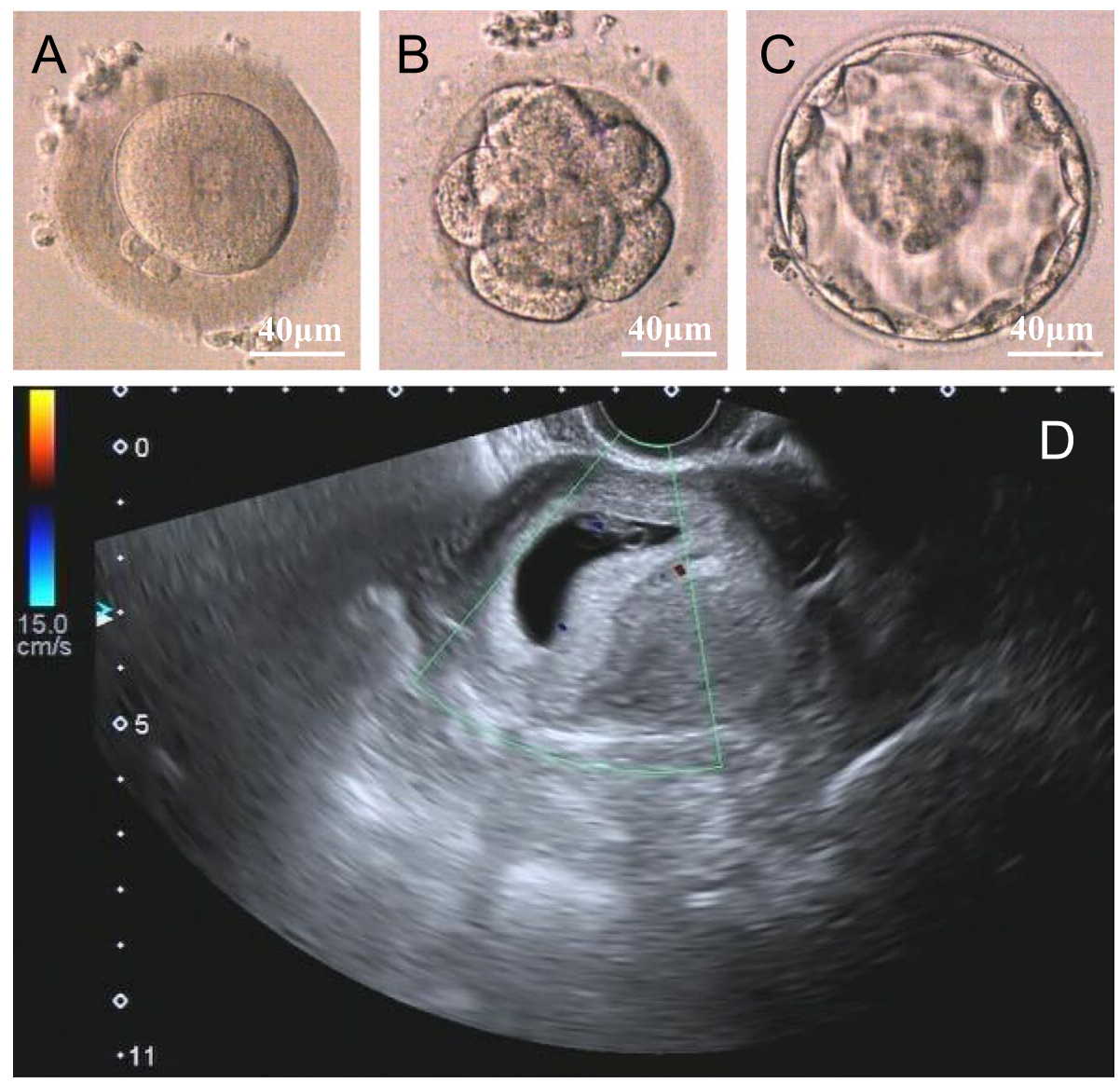

Fig. 4 Typical morphology of the implanted embryo from F1 II-1 and his partner. High-quality blastocysts formed after standard embryo culture, and F1 II-1' partner conceived successfully after the transfer of one frozen-thawed embryo. A :2 pronuclear fertilization; (B): 8-cell stage embryo; (C): blastocyst stage embryo; (D): the ultrasound image of gestational sac. Scale bar: $40 \mu \mathrm{m}$ 
SPAG6 protein was significantly lower in the spermatozoa of the two patients than in the controls, and IF analysis revealed that the fluorescent signal of SPAG6 was extremely weak and discontinuous in the sperm flagella of the patients. Taken together, these findings suggest that homozygous SPAG6 gene variants are a novel causative genetic factor leading to nonsyndromic asthenoteratozoospermia with severe MMAF.

Motile flagella and their homologous structures, cilia, share an evolutionarily conserved axonemal structure consisting of nine peripheral microtubule doublets circularly organized around a central pair of microtubules (known as the " $9+2$ " structure) [21]. Mutations in numerous genes encoding axoneme-associated proteins are closely tied to the improper assembly of cilia and flagella [6]. The human SPAG6 protein, a component of central microtubules, is essential for maintaining the structural stability of the axoneme. SPAG6 contains eight highly conserved armadillo-type repeats (ARMs), which mediate SPAG6 interactions with other central pair proteins [13]. The role of SPAG6 proteins in regulating flagellar and ciliary motility functions has been demonstrated in different biological models [14, 15, 22]. In Chlamydomonas reinhardtii, PF16, an axonemal protein orthologous to SPAG6, is localized to the central microtubule $\mathrm{C} 1$ of the axoneme, and is closely associated with flagellar motility [23]. The absence of PF16 causes instability of the $\mathrm{C} 1$ microtubule in the central pair, and flagellar paralysis [22]. Spag6-deficient mice are affected by hydrocephalus and infertility, suggesting that SPAG6 plays an important role in regulating cilial and flagellar motility [15]. Wu et al. [14] showed that SPAG6 mutations in humans lead to a multi-systemic dysfunction phenotype, including chronic respiratory tract infections and male infertility. These findings highlight the important role of SPAG6 protein in the formation of cilia and flagella.

Unlike the studies mentioned above, the two cases carrying SPAG6 homozygous variants in the present study only presented with severe asthenoteratozoospermia. Considering that no other PCD-related symptoms were found in either case, invasive operations for obtaining ciliated cells, such as brushing or mucosal biopsy from the nose or trachea, were not performed, and it was therefore impossible to examine the morphology or ultrastructure of other ciliary tissue. However, no respiratory symptoms or visceral inversion were found upon physical examination and medical consultation, which was confirmed by lung CT screening. Therefore, we speculate that partial ciliary function was preserved in the patients.

Compared with individuals carrying mutations in SPAG6 with typical PCD, the clinical phenotypes of the two probands were less severe in the present study. We speculate that this phenomenon may be attributed to a variety of factors: First, diverse mutation types and locations in some cilia-related genes may influence the severity of the phenotype in humans. For instance, the $D A N H 1$ gene is a candidate gene for PCD, which encodes a core component of inner-arm heavy chain dynein, and an investigation carried out by Sha et al. [24] demonstrated that 12 patients harboring DNAH1 variants only presented the MMAF phenotype in the absence of PCDrelated symptoms. DNAH9 is another candidate gene for PCD, but Tang et al. [25] reported that DNAH9 variants can result in non-syndromic severe asthenospermia without PCD-related symptoms. Based on this, MMAF may be another form of classical PCD [6]. Second, according to animal models, the process of flagella formation is not identical to that of cilia. For instance, the Bbs4-null mouse model failed to form sperm flagella, but developed primary cilia in other organs normally [26]. Third, gene alternative splicing is widespread in mammals, and splicing variants usually display tissue-specific expression patterns [27]. Certain variants may affect the expression of SPAG6 in the testes rather than in other tissues. In addition, there may be other microtubule proteins that are similar to SPAG6 in phylogenesis, have similar functions, and may compensate for the absence of SPAG6 in other ciliated tissue.

Assisted fertilization with ICSI technology is the preferred option for MMAF patients because of the lack of sperm motility [6]. The potential risk of genetic defects is worthy of attention, apart from sperm morphological defects. Therefore, the female partners also underwent mutation screening for SPAG6 before undergoing ICSI, and no deleterious mutations were found. After one cycle of frozen-thawed embryo transfer, the F1 II-1 couple successfully achieved clinical pregnancy. These results indicate that ICSI is an optimal management strategy for severe asthenoteratozoospermia induced by SPAG6 variants.

\section{Conclusions}

our findings expand the understanding of genetic defects in the SPAG6 gene, which is a potential pathogenic factor for syndromic severe asthenozoospermia, such as PCD, and also for non-syndromic asthenoteratozoospermia with the MMAF phenotype. ICSI is recommended as an optimal strategy with a favorable prognosis for these patients.

\section{Abbreviations}

MMAF: Multiple morphologic abnormalities of the flagella; PCD: Primary ciliary dyskinesia; WES: Whole exome sequencing; IF: Immunofluorescence; SEM: Scanning electron microscopy; TEM: Transmission electron microscopy; ICSI: Intracytoplasmic sperm injection; SIFT: Sorting Intolerant From Tolerant. 


\section{Supplementary Information}

The online version contains supplementary material available at https://doi. org/10.1186/s12958-022-00916-3.

Additional file 1: Supplementary Figure S1. Typical PCD signs was excluded based on diagnostic imaging examination in F1 II-1and F2 II-1. (A) and $\left(A^{\prime}\right)$ : The chest $X$-rays showed a normally located left-sided heart (B) and $\left(B^{\prime}\right)$ : The chest $C T$ images showed normal lung and bronchus. (C) and $\left(C^{\prime}\right)$ : The upper abdomen $C T$ images excluded visceral inversion.

\section{Additional file 2.}

Additional file 3.

Additional file 4.

Additional file 5.

\section{Acknowledgements}

Not applicable

\section{Authors' contributions}

CX, DT, ML, XH and YC were responsible for conception of the study. $H G, Y G$, $\mathrm{QT}, \mathrm{KL}$, and $\mathrm{CW}$ were in charge of data collection and clinical assessment. $\mathrm{CX}, \mathrm{DT}, \mathrm{ML}, \mathrm{ZS}$, and GW performed the experiments. DT and $\mathrm{KL}$ analyzed the data. CX, DT, and YG wrote the draft. HW, GL, ML, and XH revised the draft. All authors read and approved the final manuscript.

\section{Funding}

This study was supported by the National Natural Science Foundation of China (grant numbers 8210061870, 81971441 and 82071705), and the National Key R\&D Program of China (No. 2021 YFC2700901).

\section{Availability of data and materials}

The datasets utilized and/or analyzed in the study are available from the corresponding author on reasonable request.

\section{Declarations}

\section{Ethics approval and consent to participate}

This study was reviewed and approved by the Ethics Committee of the First Affiliated Hospital of Anhui Medical University.

\section{Consent for publication}

All the participants signed written informed consents for this study.

\section{Competing interests}

The authors declare that they have no competing interests.

\section{Author details}

${ }^{1}$ Reproductive Medicine Center, Department of Obstetrics and Gynecology, the First Affiliated Hospital of Anhui Medical University, No 218 Jixi Road, Hefei 230022, Anhui, China. ${ }^{2}$ NHC Key Laboratory of Study On Abnormal Gametes and Reproductive Tract (Anhui Medical University), No 81 Meishan Road, Hefei 230032, Anhui, China. ${ }^{3}$ Key Laboratory of Population Health Across Life Cycle (Anhui Medical University), Ministry of Education of the People's Republic of China, No 81 Meishan Road, Hefei 230032, Anhui, China. ${ }^{4}$ Anhui Province Key Laboratory of Reproductive Health and Genetics, No 81 Meishan Road, Hefei 230032, Anhui, China. ${ }^{5}$ Biopreservation and Artificial Organs, Anhui Provincial Engineering Research Center, Anhui Medical University, No 81 Meishan Road, Hefei 230032, Anhui, China.

Received: 18 October 2021 Accepted: 21 February 2022 Published: 1 March 2022

\section{References}

1. Vander Borght M, Wyns C. Fertility and infertility: Definition and epidemiology. Clin Biochem. 2018;62:2-10.
2. Sun H, Gong TT, Jiang YT, Zhang S, Zhao YH, Wu QJ. Global, regional, and national prevalence and disability-adjusted life-years for infertility in 195 countries and territories, 1990-2017: results from a global burden of disease study, 2017. Aging (Albany NY). 2019;11:10952-91.

3. Agarwal A, Mulgund A, Hamada A, Chyatte MR. A unique view on male infertility around the globe. Reprod Biol Endocrinol. 2015;13:37.

4. Krausz C, Riera-Escamilla A. Genetics of male infertility. Nat Rev Urol. 2018;15:369-84.

5. Ben Khelifa M, Coutton C, Zouari R, Karaouzene T, Rendu J, Bidart M, Yassine S, Pierre V, Delaroche J, Hennebicq S, Grunwald D, Escalier D, Pernet-Gallay K, Jouk PS, Thierry-Mieg N, Toure A, Arnoult C, Ray PF. Mutations in DNAH1, which encodes an inner arm heavy chain dynein, lead to male infertility from multiple morphological abnormalities of the sperm flagella. Am J Hum Genet. 2014;94:95-104.

6. Coutton C, Escoffier J, Martinez G, Arnoult C, Ray PF. Teratozoospermia: spotlight on the main genetic actors in the human. Hum Reprod Update. 2015;21:455-85.

7. Liu C, Miyata H, Gao Y, Sha Y, Tang S, Xu Z, Whitfield M, Patrat C, Wu H, Dulioust E, Tian S, Shimada K, Cong J, Noda T, Li H, Morohoshi A, Cazin C, Kherraf ZE, Arnoult C, Jin L, He X, Ray PF, Cao Y, Toure A, Zhang F, Ikawa M. Bi-allelic DNAH8 Variants Lead to Multiple Morphological Abnormalities of the Sperm Flagella and Primary Male Infertility. Am J Hum Genet. 2020;107:330-41.

8. Liu C, Lv M, He X, Zhu Y, Amiri-Yekta A, Li W, Wu H, Kherraf ZE, Liu W, Zhang J, Tan Q, Tang S, Zhu YJ, Zhong Y, Li C, Tian S, Zhang Z, Jin L, Ray P, Zhang F, Cao Y. Homozygous mutations in SPEF2 induce multiple morphological abnormalities of the sperm flagella and male infertility. J Med Genet. 2020;57:31-7.

9. Coutton C, Martinez G, Kherraf ZE, Amiri-Yekta A, Boguenet M, Saut A, He X, Zhang F, Cristou-Kent M, Escoffier J, Bidart M, Satre V, Conne B, FouratiBen Mustapha S, Halouani L, Marrakchi O, Makni M, Latrous H, Kharouf M, Pernet-Gallay K, Bonhivers M, Hennebicq S, Rives N, Dulioust E, Toure A, Gourabi H, Cao Y, Zouari R, Hosseini SH, Nef S, Thierry-Mieg N, Arnoult C, Ray PF. Bi-allelic Mutations in ARMC2 Lead to Severe Astheno-Teratozoospermia Due to Sperm Flagellum Malformations in Humans and Mice. Am J Hum Genet. 2019;104:331-40.

10. Ni X, Wang J, Lv M, Liu C, Zhong Y, Tian S, Wu H, Cheng H, Gao Y, Tan Q, Chen B, Li Q, Song B, Wei Z, Zhou P, He X, Zhang F, Cao Y. A novel homozygous mutation in WDR19 induces disorganization of microtubules in sperm flagella and nonsyndromic asthenoteratospermia. J Assist Reprod Genet. 2020;37:1431-9.

11. Lores P, Dacheux D, Kherraf ZE, NsotaMbango JF, Coutton C, Stouvenel L, laly-Radio C, Amiri-Yekta A, Whitfield M, Schmitt A, Cazin C, Givelet M, Ferreux L, FouratiBen Mustapha S, Halouani L, Marrakchi O, Daneshipour A, El Khouri E, Do Cruzeiro M, Favier M, Guillonneau F, Chaudhry M, Sakheli Z, Wolf JP, Patrat C, Gacon G, Savinov SN, Hosseini SH, Robinson DR, Zouari R, Ziyyat A, Arnoult C, Dulioust E, Bonhivers M, Ray PF, Toure A. Mutations in TTC29, Encoding an Evolutionarily Conserved Axonemal Protein, Result in Asthenozoospermia and Male Infertility. Am J Hum Genet. 2019;105:1148-67.

12. Liu C, He X, Liu W, Yang S, Wang L, Li W, Wu H, Tang S, Ni X, Wang J, Gao Y, Tian S, Zhang L, Cong J, Zhang Z, Tan Q, Zhang J, Li H, Zhong Y, Lv M, Li J, Jin L, Cao Y, Zhang F. Bi-allelic Mutations in TTC29 Cause Male Subfertility with Asthenoteratospermia in Humans and Mice. Am J Hum Genet. 2019;105:1168-81.

13. Neilson LI, Schneider PA, Van Deerlin PG, Kiriakidou M, Driscoll DA, Pellegrini MC, Millinder S, Yamamoto KK, French CK, Strauss JF 3rd. cDNA cloning and characterization of a human sperm antigen (SPAG6) with homology to the product of the Chlamydomonas PF16 locus. Genomics. 1999:60:272-80.

14. Wu H, Wang J, Cheng H, Gao Y, Liu W, Zhang Z, Jiang H, Li W, Zhu F, LV M, Liu C, Tan Q, Zhang X, Wang C, Ni X, Chen Y, Song B, Zhou P, Wei Z, Zhang F, He X, Cao Y. Patients with severe asthenoteratospermia carrying SPAG6 or RSPH3 mutations have a positive pregnancy outcome following intracytoplasmic sperm injection. J Assist Reprod Genet. 2020;37:829-40.

15. Sapiro R, Kostetskii I, Olds-Clarke P, Gerton GL, Radice GL, Strauss IJ. Male infertility, impaired sperm motility, and hydrocephalus in mice deficient in sperm-associated antigen 6. Mol Cell Biol. 2002;22:6298-305.

16. Tang S, Wang X, Li W, Yang X, Li Z, Liu W, Li C, Zhu Z, Wang L, Wang J, Zhang L, Sun X, Zhi E, Wang H, Li H, Jin L, Luo Y, Wang J, Yang S, Zhang F. Biallelic Mutations in CFAP43 and CFAP44 Cause Male Infertility with 
Multiple Morphological Abnormalities of the Sperm Flagella. Am J Hum Genet. 2017;100:854-64.

17. Cooper TG, Noonan E, von Eckardstein S, Auger J, Baker HW, Behre HM, Haugen TB, Kruger T, Wang C, Mbizvo MT, Vogelsong KM. World Health Organization reference values for human semen characteristics. Hum Reprod Update. 2010;16:231-45.

18. Lv M, Liu W, Chi W, Ni X, Wang J, Cheng H, Li WY, Yang S, Wu H, Zhang J, Gao Y, Liu C, Li C, Yang C, Tan Q, Tang D, Zhang J, Song B, Chen YJ, Li Q, Zhong Y, Zhang Z, Saiyin H, Jin L, Xu Y, Zhou P, Wei Z, Zhang C, He X, Zhang F, Cao Y. Homozygous mutations in DZIP1 can induce asthenoteratospermia with severe MMAF. J Med Genet. 2020;57:445-53.

19. He X, Li W, Wu H, Lv M, Liu W, Liu C, Zhu F, Li C, Fang Y, Yang C, Cheng H, Zhang J, Tan J, Chen T, Tang D, Song B, Wang X, Zha X, Wang H, Wei Z, Yang S, Saiyin H, Zhou P, Jin L, Wang J, Zhang Z, Zhang F, Cao Y. Novel homozygous CFAP69 mutations in humans and mice cause severe asthenoteratospermia with multiple morphological abnormalities of the sperm flagella. J Med Genet. 2019;56:96-103.

20. Zhu F, Liu C, Wang F, Yang X, Zhang J, Wu H, Zhang Z, He X, Zhang Z, Zhou P, Wei Z, Shang Y, Wang L, Zhang R, Ouyang YC, Sun QY, Cao Y, Li W. Mutations in PMFBP1 Cause Acephalic Spermatozoa Syndrome. Am J Hum Genet. 2018;103:188-99.

21 Ishikawa T. Axoneme Structure from Motile Cilia. Cold Spring Harb Perspect Biol. 2017;9(1):a028076.

22. Adams GM, Huang B, Piperno G, Luck DJ. Central-pair microtubular complex of Chlamydomonas flagella: polypeptide composition as revealed by analysis of mutants. J Cell Biol. 1981;91:69-76.

23. Smith EF, Lefebvre PA. PF16 encodes a protein with armadillo repeats and localizes to a single microtubule of the central apparatus in Chlamydomonas flagella. J Cell Biol. 1996;132:359-70.

24. Sha Y, Yang $X$, Mei L, Ji Z, Wang X, Ding L, Li P, Yang S. DNAH1 gene mutations and their potential association with dysplasia of the sperm fibrous sheath and infertility in the Han Chinese population. Fertil Steril. 2017;107(1312-8):e2.

25. Tang D, Sha Y, Gao Y, Zhang J, Cheng H, Zhang J, Ni X, Wang C, Xu C, Geng $\mathrm{H}, \mathrm{He}$ X, Cao Y. Novel variants in DNAH9 lead to nonsyndromic severe asthenozoospermia. Reprod Biol Endocrinol. 2021;19:27.

26. Mykytyn K, Mullins RF, Andrews M, Chiang AP, Swiderski RE, Yang B, Braun T, Casavant T, Stone EM, Sheffield VC. Bardet-Biedl syndrome type 4 (BBS4)-null mice implicate Bbs4 in flagella formation but not global cilia assembly. Proc Natl Acad Sci U S A. 2004;101:8664-9.

27. Yu Y, Fuscoe JC, Zhao C, Guo C, Jia M, Qing T, Bannon DI, Lancashire L, Bao W, Du T, Luo H, Su Z, Jones WD, Moland CL, Branham WS, Qian F, Ning B, Li Y, Hong H, Guo L, Mei N, Shi T, Wang KY, Wolfinger RD, Nikolsky Y, Walker SJ, Duerksen-Hughes P, Mason CE, Tong W, Thierry-Mieg J, Thierry-Mieg D, Shi L, Wang C. A rat RNA-Seq transcriptomic BodyMap across 11 organs and 4 developmental stages. Nat Commun. 2014;5:3230.

\section{Publisher's Note}

Springer Nature remains neutral with regard to jurisdictional claims in published maps and institutional affiliations.

Ready to submit your research? Choose BMC and benefit from:

- fast, convenient online submission

- thorough peer review by experienced researchers in your field

- rapid publication on acceptance

- support for research data, including large and complex data types

- gold Open Access which fosters wider collaboration and increased citations

- maximum visibility for your research: over $100 \mathrm{M}$ website views per year

At BMC, research is always in progress.

Learn more biomedcentral.com/submissions 